\title{
Imágenes del depósito de gadolinio en el sistema nervioso central
}

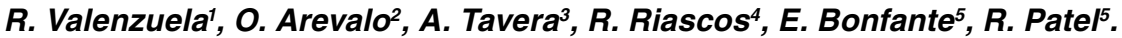

1. Neurorradiólogo, Center of Advanced Imaging Processing, The University of Texas Health Science Center at Houston, Huston, TX, USA.

2. Radiólogo, Departamento de Imagenología Diagnóstica e Intervencional, Hospital Universitario Nacional de Colombia, Bogotá- Colombia.

3. Interno de medicina, Universidad El Bosque, Bogotá- Colombia.

4. Profesor Asociado, Diagnostic and interventional imaging at The University of Texas Health Science Center at Houston, Huston, TX, USA.

5. Profesor Asociado, Diagnostic and interventional imaging at The University of Texas Health Science Center at Houston, Huston, TX, USA.

\section{Images of gadolinio deposit in the central nervous system}

\begin{abstract}
Magnetic resonance imaging has been shown to be very useful in the diagnosis and monitoring of multiple diseases affecting the central nervous system. Gadolinium has been used extensively worldwide. It has been estimated that since its introduction more than two hundred million doses of GBCA have been administered globally.

Gadolinium-based contrast agents (GBCAs) were long considered medicines with a high safety profile, the literature reports incidences of immediate adverse effects such as headache, dizziness, and altered sense of taste. Studies performed a few years after the introduction of GBCA showed that there were gadolinium deposits in the tissues but their clinical significance was not known. It wasn't until 2006 when the first reports were published that associated the gadolinium ion tissue deposits from GBCA with a systemic inflammatory entity of the connective tissue similar to scleroderma known as Nephrogenic Systemic Fibrosis (NSF), in patients with chronic renal failure. In 2013, the association between the use of GBCAs and the progressive increase in the signal intensity of the dentate nucleus and the globus pallidus in T1-weighted MRI images without contrast medium, was described for the first time.

This review describes the most relevant aspects of the pathophysiology of these findings taking into account their differential diagnosis.

Key words: Gadolinium deposits, nephrogenic systemic fibrosis, central nervous system.
\end{abstract}

\section{Resumen}

Las imágenes por resonancia magnética han mostrado ser de gran utilidad en el diagnóstico y seguimiento de múltiples enfermedades que afectan el sistema nervioso central. El gadolinio se ha utilizado ampliamente a nivel mundial. Se estima que desde su introducción se han administrado más de doscientos millones de dosis de $M C B G$ en el mundo.

Los medios de contraste basados en Gadolinio (MCBG) fueron considerados por mucho tiempo medicamentos con un alto perfil de seguridad, la literatura reporta incidencia de efectos adversos inmediatos como cefalea, mareo y alteración del sentido del gusto. Estudios realizados pocos años después de la introducción de los MCBG mostraron que había depósitos de gadolinio en los tejidos, pero no se conocía su significancia clínica Fue hasta 2006 cuando se publicaron los primeros reportes que asociaban el depósito tisular del ion gadolinio proveniente de los MCBG con una entidad inflamatoria sistémica del tejido conectivo similar a la escleroderma conocida como Fibrosis Sistémica Nefrogénica (FSN) en pacientes con falla renal crónica.

En 2013 se describió por primera vez la asociación entre el uso de los MCBG y el aumento progresivo de la intensidad de señal del núcleo dentado y los globos pálidos en las imágenes de RM ponderadas en T1 sin medio de contraste.

En esta revisión se describen los aspectos más relevantes de la fisiopatología de estos hallazgos considerando su diagnóstico diferencial.

Palabras claves: Depósitos de Gadolinio, fibrosis sistémica nefrogénica, sistema nervioso central, resonancia magnética. 
Valenzuela R., et al. Imágenes del depósito de gadolinio en el sistema nervioso central. Rev Chil Radiol 2017; 23(2): 59-65.

Correspondencia: Raúl Valenzuela / raul.f.valenzuela@uth.tmc.edu.

Trabajo recibido el 7 de marzo de 2017. Aceptado para publicación el 23 de julio de 2017.

\section{Introducción}

Las imágenes por resonancia magnética (RM) han mostrado ser de gran utilidad en el diagnóstico y seguimiento de múltiples enfermedades que afectan el sistema nervioso central (SNC) por su alta resolución espacial, consistencia, reproducibilidad y disponibilidad. En algunas ocasiones es necesario administrar al paciente compuestos químicos llamados medios de contraste que mejoran la visualización y caracterización de las diferentes lesiones en RM. Los medios de contraste basados en Gadolinio (MCBG) son los compuestos químicos que se utilizan con mayor frecuencia en la práctica clínica diaria'. Dependiendo de la vía de administración los MCBG se acumulan de forma transitoria en ciertos tejidos normales o anormales, este fenómeno resulta en realce en las imágenes de RM por la propiedad física de los MCBG de acortar el tiempo de relajación $\mathrm{T}^{2,3}$. Al ser administrado por vía intravenosa, los MCBG condicionan realce en las zonas del SNC en las que existe mayor permeabilidad de la barrera hematoencefálica (BHE), bien sea por variantes anatómicas normales o por la presencia de un proceso patológico ${ }^{2}$. El primer MCBG aprobado por la Administración de Comida y Medicamentos de los Estados Unidos (FDA por sus siglas en inglés) fue el Gadopentetato de dimeglumina (Magnevist ${ }^{\circledR}$ ) en $1988^{4}$ y se estima que desde entonces se han administrado más de doscientos millones de dosis de MCBG en el mundo.

Los MCBG fueron considerados por mucho tiempo medicamentos con un alto perfil de seguridad, la literatura reporta incidencia de efectos adversos inmediatos como cefalea, mareo y alteración del sentido del gusto en sólo el $0,03 \%$, los cuales son más prevalentes en pacientes atópicos, asmáticos o con una tasa de infusión rápida ${ }^{5}$. Estudios realizados pocos años después de la introducción de los MCBG mostraron que había depósitos de gadolinio en los tejidos, pero no se conocía su significancia clínica ${ }^{6}$. Fue hasta 2006 cuando se publicaron los primeros reportes que asociaban el depósito tisular del ion gadolinio proveniente de los MCBG con una entidad inflamatoria sistémica del tejido conectivo similar a la escleroderma conocida como Fibrosis Sistémica Nefrogénica (FSN) en pacientes con falla renal cróni$\mathrm{Ca}^{7-9}$. Dado el riesgo de FSN el Colegio Americano de Radiología publicó nuevas guías para el uso seguro de los MCBG, y luego de restringir el uso de estos medicamentos en los pacientes con disminución de la Tasa de Filtración Glomerular (TFG) su incidencia ha disminuido dramáticamente ${ }^{5}$.

En 2013 Kanda y cols $^{10}$ describieron por primera vez la asociación entre el uso de los MCBG y el aumento progresivo de la intensidad de señal del núcleo dentado y los globos pálidos en las imágenes de RM ponderadas en T1 sin medio de contraste; según los autores esos hallazgos indican depósito progresivo de gadolinio en el tejido neural. Desde entonces se han publicado múltiples estudios con respecto al deposito de gadolinio en el tejido neural. A continuación, se presenta una corta revisión de los aspectos más relevantes de la fisiopatología de estos hallazgos.

\section{Gadolinio}

El gadolinio (Gd) es un metal pesado de la familia de los lantánidos o tierras raras, con numero atómico 641. Los metales pesados como el Gd son tóxicos para los mamíferos al interferir en múltiples vías metabólicas celulares, sus efectos biológicos más conocidos son:

- Similitud con el Calcio $(\mathrm{Ca})^{3}$ : El estado de oxidación más común del gadolinio es $\mathrm{Gd}^{3}+$ y en ese estado su diámetro es similar al del ion $\mathrm{Ca}^{2+}$, razón por la cual compite por sus sitios de unión en las moléculas dependientes de Ca como los receptores de membrana celular, la cascada de la coagulación, la matriz mineral ósea, entre otros ${ }^{4}$.

- No es soluble en un medio acuoso con pH fisiológico (7.4), razón por la que se precipita en los tejidos y su tasa de eliminación es del orden de 1 a $3 \%$ por día ${ }^{3}$. Los depósitos de Gd siguen el mismo patrón de los depósitos de sales de calcio en los tejidos, por ejemplo, el hueso, los riñones y los núcleos grises en el SNC. No es claro si ese depósito ocurre por difusión pasiva o por vías metabólicas activas ${ }^{1}$.

Luego, se presenta una corta revisión de los aspectos más relevantes de la fisiopatología de estos hallazgos. Los agentes quelantes son compuestos orgánicos que se unen a los metales pesados para disminuir sus efectos tóxicos en el organismo. Los medios de contraste basados en gadolinio (MCBG) son moléculas compuestas por un agente quelante y $\mathrm{Gd}$, lo que le confiere propiedades farmacocinéticas que facilitan su administración, metabolismo y eliminación y disminuye de forma significativa su 
toxicidad, sus interacciones biológicas y su depósito en los tejidos ${ }^{1,3,4}$. La estabilidad de un MCBG hace referencia a la tasa de disociación entre el agente quelante y el Gd3+ con respecto al tiempo. Esa disociación ocurre espontáneamente obedeciendo a las leyes de la termodinámica y de la interacción de la materia, sin embargo su $\mathrm{t} 1 / 2$ puede ser modificado por factores intrínsecos como el tipo de agente quelante o extrínsecos como la presencia de enzimas, la temperatura y el $\mathrm{pH}^{1,3,4}$. En términos prácticos, a mayor estabilidad menor probabilidad que un MCBG libere $\mathrm{Gd}^{3+}$ tóxico en el organismo.

\section{Medios de contraste basados en gadolinio (MCBG)}

A la fecha la FDA ha aprobado nueve moléculas para el uso en el ámbito clínico ${ }^{2,13}$. Los MCBG se clasifican con base en el tipo de agente quelante en lineales y macrocíclicos, que a su vez se subdividen en iónicos y no iónicos dependiendo de si dichas moléculas tienen carga o son apolares ${ }^{1}$. Los MCBG más estables son los macrocíclicos y los iónicos, ya que la estructura tridimensional de los macrocíclicos y las interacciones de carga en los iónicos previenen la disociación del $\mathrm{Gd}^{3+}$ y el agente quelante ${ }^{1,13}$. Los MCBG lineales iónicos son los más utilizados en la práctica clínica e incluyen el Gd-DTPA (Magnevist ${ }^{\circledR}$ ), Gd-BOPTA (MultiHance ${ }^{\circledR}$ ), Gd-EOB-DTPA (Primovist ${ }^{\circledR} /$ Eovist $\AA$ ) y MS-325 (Vasovist $\AA /$ Ablavar $\AA$ ); por otra parte, los lineales no iónicos son Gd-DTPA-BMA (Omniscan®) y Gd-DTPA-BMEA (OptiMARK®). El único MCBG macrocíclico iónico es el Gd-DOTA (Dotarem ${ }^{\circledR}$ ) y los no iónicos son Gd-HP-DO3A (ProHance ${ }^{\circledR}$ ) y Gd-BT-DO3A (Gadovist $\AA /$ Gadavist $\left.{ }^{\circledR}\right)^{1}$ (Tabla 1) .

Hallazgos en RM del depósito de gadolinio en el sistema nervioso central.

En el año 2013 Kanda y cols ${ }^{10}$. describieron el aumento progresivo de la intensidad de señal del núcleo dentado (ND) y del globo pálido (GP) en las imágenes de RM ponderadas en T1 sin medio de contraste y comparado con el número de dosis previas de MCBG encontrando una correlación estadísticamente significativa; por esta razón, aunque la naturaleza de esos hallazgos en imágenes no se estableció de forma exacta, el depósito de gadolinio se incluyó en la lista de los posibles diagnósticos diferenciales junto con interpretaciones clásicas de dichas alteraciones como los cambios por radioterapia y la esclerosis múltiple, entre otros. En su trabajo inicial, Kanda ${ }^{10}$ sugiere que el aumento de la intensidad de señal del ND y del GP en los pacientes con enfermedades crónicas del SNC corresponde a depósito tisular de gadolinio secundario a las múltiples dosis de MCBG y no al tratamiento utilizado o a la historia natural de la enfermedad. Esa teoría fue soportada por el estudio de Quattrocchi y $\operatorname{cols}^{14}$. quienes evaluaron las resonancias magnéticas de seguimiento a pacientes con meningiomas en manejo conservador con protocolo de observación sin ningún tipo de intervención terapéutica encontrando los mismos cambios progresivos del ND y GP.

En $2015 \mathrm{McDonald}$ y cols ${ }^{15}$. publicaron un estudio en el cual usaron la técnica de "Espectrometría de masas con plasma acoplado inductivamente" (ICP-MS por sus siglas en inglés) para cuantificar el contenido de Gadolinio en muestras de tejido cerebral de pacientes con función renal normal. Las muestras de tejido cerebral se obtuvieron de autopsias de 13 pacientes sometidos al menos a cuatro resonancias con MCBG en los últimos 14 años y fueron comparadas con muestras obtenidas de 10 pacientes que nunca se expusieron a estos medicamentos. Este estudio no encontró gadolinio en las muestras de los pacientes que no fueron expuestos a los MCBG; por otra parte, en los pacientes expuestos se encontraron niveles cuantificables en el endotelio capilar (2/3) y

Tabla 1. Clasificación de los medios de contraste basados en gadolinio.

\begin{tabular}{|c|c|c|c|c|}
\hline $\begin{array}{l}\text { Estructura del } \\
\text { agente quelante }\end{array}$ & $\begin{array}{l}\text { lónico vs. } \\
\text { No iónico }\end{array}$ & $\begin{array}{l}\text { Compuesto } \\
\text { químico }\end{array}$ & $\begin{array}{l}\text { Nombre } \\
\text { genérico }\end{array}$ & $\begin{array}{l}\text { Nombre } \\
\text { comercial }\end{array}$ \\
\hline Lineales & lónico & $\begin{array}{l}\text { Gd-DTPA } \\
\text { Gd-BOPTA } \\
\text { Gd-EOB-DTPA } \\
\text { MS-325 }\end{array}$ & $\begin{array}{l}\text { Gadopentetato de dimeglumina } \\
\text { Gadobenato de dimeglumina } \\
\text { Gadoxetato disódico } \\
\text { Gadofosveset trisódico }\end{array}$ & $\begin{array}{l}\text { Magnevist } ₫ \\
\text { MultiHance } ₫ \\
\text { Primovist } ₫ / \text { Eovist } \circledast \\
\text { Vasovist } ₫ / \text { Ablavar } \circledast\end{array}$ \\
\hline & No iónico & $\begin{array}{l}\text { Gd-DTPA-BMA } \\
\text { Gd-DTPA-BMEA }\end{array}$ & $\begin{array}{l}\text { Gadodiamida } \\
\text { Gadoversetamida }\end{array}$ & $\begin{array}{l}\text { Omniscan® } \\
\text { OptiMARK® }\end{array}$ \\
\hline Macrocíclicos & $\begin{array}{l}\text { lónico } \\
\text { No iónico }\end{array}$ & $\begin{array}{l}\text { Gd-DOTA } \\
\text { Gd-HP-DO3A } \\
\text { Gd-BT-DO3A }\end{array}$ & $\begin{array}{l}\text { Gadoterato de meglumina } \\
\text { Gadoteridol } \\
\text { Gadobutrol }\end{array}$ & $\begin{array}{l}\text { Dotarem } \AA \\
\text { ProHance } \AA \\
\text { Gadovist } \AA / \text { Gadavist } \AA\end{array}$ \\
\hline
\end{tabular}


en el intersticio del tejido nervioso (1/3) del núcleo dentado, puente, globo pálido y tálamo, siendo mayor la concentración en el núcleo dentado ${ }^{15}$. El gadolinio que se encontró en el intersticio del SNC no se asociaba con alteraciones de la barrera hematoencefálica $(\mathrm{BHE})$ ni de la arquitectura normal del tejido en el estudio histológico. Teniendo en cuenta el estricto control que ejerce la barrera hematoencefálica en el tránsito de sustancias desde el torrente sanguíneo hacia el parénquima del SNC se han propuesto dos mecanismos para explicar el depósito de gadolinio en tejidos con BHE intacta ${ }^{3,4}$ :

- Transmetalación: Es el intercambio de un metal como el $\mathrm{Gd}^{3+}$ proveniente de un MCBG por otro ion de similares características estructurales como el $\mathrm{Zn}^{2+}, \mathrm{Mg}^{2+}, \mathrm{Ca}^{2+}, \mathrm{Fe}^{3+}$ de una macromolécula implicada en determinadas vías metabólicas.

- Difusión del $\mathrm{Gd}^{3+}$ como ion libre luego de disociarse del agente quelante de un MCBG inestable.

Kanda y cols $^{16}$. demostraron que el gadolinio también se deposita en otras áreas del sistema nervioso central como la corteza frontal, la sustancia blanca frontal, la corteza cerebelosa, y la sustancia blanca cerebelosa. Estos datos sugieren que los hallazgos en imágenes pueden subestimar la extensión de los depósitos de gadolinio, ya que solamente se pueden identificar cambios de la intensidad de señal de una estructura cuando la concentración tisular excede $30 \mu \mathrm{mol} / \mathrm{L}$ de gadolinio quelado ${ }^{5}$. Aún no ha sido posible definir con exactitud si los depósitos tisulares corresponden exclusivamente al gadolinio en su forma iónica o al MCBG en su totalidad, incluyendo el agente quelante ${ }^{17}$.

Estudios posteriores demuestran que los cambios de la intensidad de señal en RM del ND y del GP se asocian casi invariablemente con el uso de los MCBG lineales, en particular con Gadodiamida ${ }^{14,18}$ y menos frecuentemente con Gadopentetato de dimeglumina ${ }^{4}$. Robert y cols $^{19}$ condujeron un estudio en ratas que fueron expuestas a diferentes MCBG lineales y macrocíclicos y concluyeron que los cambios de intensidad en RM secundarios al depósito de gadolinio fueron exclusivos de las moléculas lineales. Se considera que los depósitos de gadolinio son proporcionales al número de dosis y se asocian al uso de MCBG lineales debido a su menor estabilidad y a la mayor tasa de disociación con respecto a los MCBG macrocíclicos ${ }^{12,17-20}$. En julio de 2016 Murata y cols ${ }^{21}$ publicaron los resultados preliminares de un estudio en el que realizan mediciones cuantitativas de los niveles de gadolinio con ICP-MS en tejido del SNC obtenido de autopsias de pacientes con función renal normal que fueron expuestos tanto a MCBG lineales (Gadobenato de dimeglumina y Gadoxetato disódico) como a MCBG macrocíclicos (Gadoteridol y Gadobutrol). Los resultados reportados indican que también hay depósito tisular de gadolinio con los MCBG macrocíclicos aunque en una concentración 20 veces menor comparado con los lineales, lo que explicaría porqué no se identifican cambios en las imágenes de $\mathrm{RM}^{20,21}$.

Los estudios realizados en la FSN mostraron que la disminución de la tasa de filtración glomerular (TFG) en los pacientes con falla renal favorece el efecto tóxico del gadolinio al aumentar el tiempo medio de eliminación de los MCBG; esto dado que la tasa de disociación de los MCBG es en función del tiempo, a mayor tiempo de eliminación mayor probabilidad de separación del $\mathrm{Gd}^{3+}$ de su agente quelante ${ }^{4,9}$. Sin embargo, se ha encontrado que aunque el depósito tisular es más severo en pacientes con falla renal ${ }^{22}$, éste también ocurre en pacientes con función renal intacta ${ }^{3,15,21}$, en quienes aparece en promedio después de la quinta dosis ${ }^{23}$. No se han encontrado diferencias significativas en el comportamiento de los depósitos de gadolinio en la población pediátrica ${ }^{24,25}$.

EI MCBG con mayor incidencia de depósito en el ND y el GB es el lineal no iónico Gadodiamida (Omniscan $\left.{ }^{\circledR}\right)$, seguido en menor proporción por los lineales iónicos Gadopentetato de dimeglumina (Magnevist ${ }^{\circledR}$ ) y Gadobenato de dimeglumina (MultiHance $\left.{ }^{\circledR}\right)^{3}$. Al momento no se ha determinado la importancia clínica del depósito de gadolinio en las estructuras del sistema nervioso central. ya que los pacientes estudiados no presentan síntomas relacionados con disfunción de las áreas afectadas ${ }^{1,3,5,14}$.

\section{Diagnóstico diferencial}

El diagnóstico diferencial de la hiperintensidad del ND y del GP en las imágenes ponderadas en T1 sin medio de contraste es amplio e incluye enfermedades metabólicas, neurofibromatosis, depósitos de otros metales y cambios por la edad, entre otros ${ }^{26,27}$ (Tabla 2).

\section{Conclusiones}

El uso de medios de contraste basados en gadolinio (MCBG) en las imágenes por resonancia magnética ha permitido avances significativos en el diagnóstico y tratamiento de múltiples enfermedades en el último cuarto de siglo. A pesar de ser considerados medicamentos con alto nivel de seguridad, los MCBG se han asociado a desenlaces adversos tales como la fibrosis sistémica nefrogénica y depósito en el tejido neural, entre otros. El gadolinio es un metal tóxico para diferentes procesos bioquímicos celulares, por esa razón es administrado en conjunto con un agente quelante que impide la liberación de su forma ionizada tóxica y facilita su administración, distribución y eliminación del organismo. Los MCBG se clasifican según el tipo de agente quelante en lineales y macrocíclicos, y según su carga en iónicos 
Tabla 2. Diagnóstico diferencial de la hiperintensidad del núcleo dentado en resonancia magnética ponderada en $\mathrm{T} 1$ sin medio de contraste.

\begin{tabular}{|l|l|}
\hline Fisiológica & Calcificación por la edad \\
\hline Enfermedades infiltrativas & $\begin{array}{l}\text { Histiocitosis de células de Langerhans } \\
\text { Enfermedad de Erdheim-Chester }\end{array}$ \\
\hline Enfermedades hereditarias & \begin{tabular}{l} 
Neurofibromatosis tipo 1 \\
\hline Depósito de otros metales
\end{tabular} \\
& $\begin{array}{l}\text { Calcio (Síndrome de Fahr) } \\
\text { Manganeso } \\
\text { Hierro } \\
\text { Cobre } \\
\text { Mercurio }\end{array}$ \\
\hline
\end{tabular}

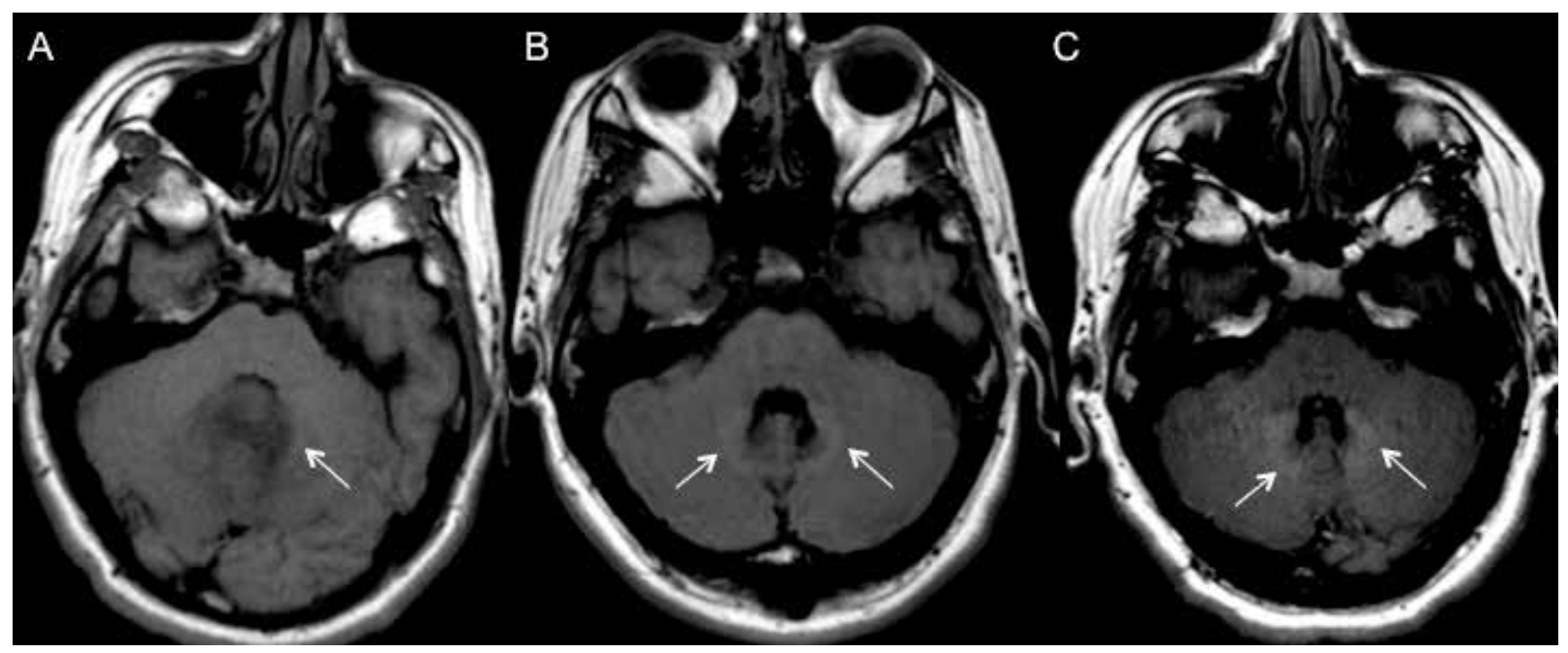

Figura 1. Imagen de resonancia magnética, T1, cortes axiales. Paciente en tratamiento por Hemangioblastoma cerebeloso en quien se evidencia reforzamiento del núcleo dentado (flechas) progresivo a través del tiempo, en relación con la toma de nuevos estudios con contraste. A) noviembre 2011, B) agosto 2013, C) noviembre 2016.

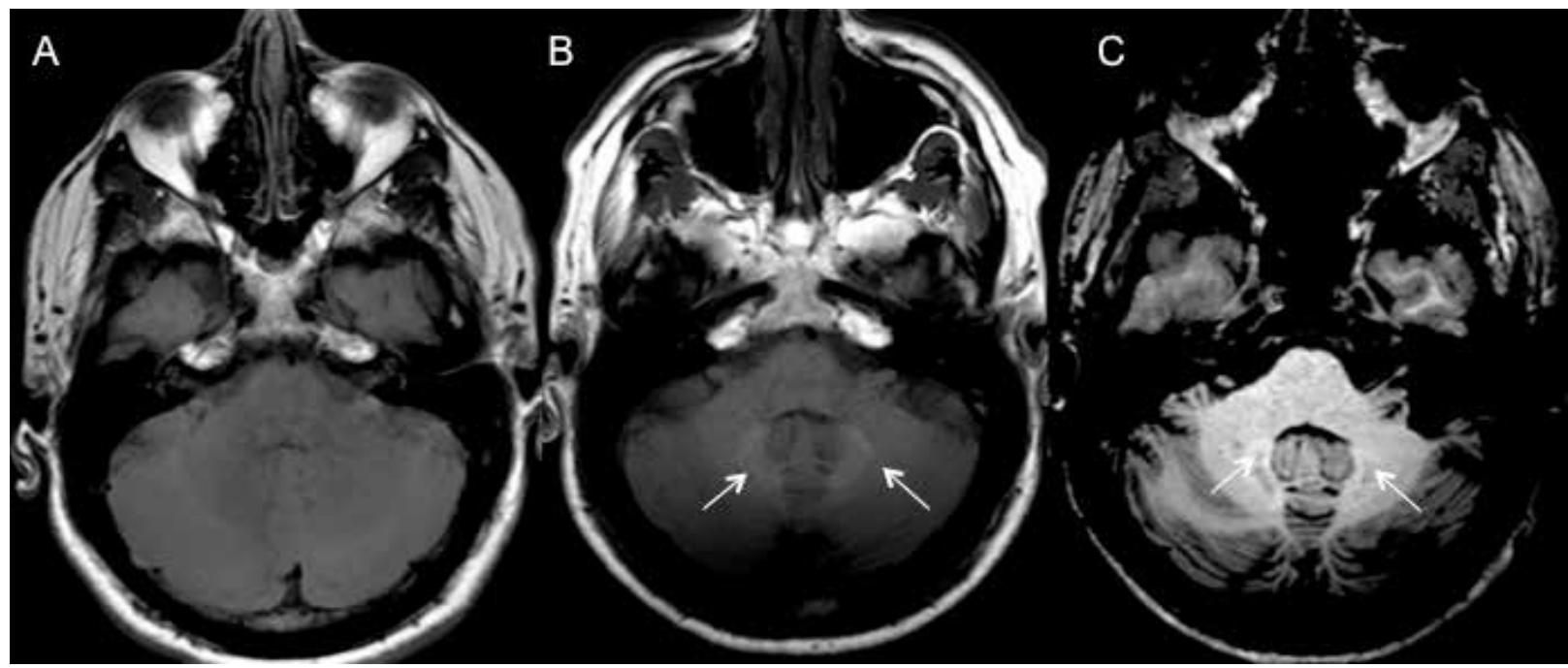

Figura 2. Imagen de resonancia magnética, T1, cortes axiales. Paciente en seguimiento por Glioblastoma, en quien se evidencia reforzamiento del núcleo dentado (flechas) progresivo a través del tiempo, en relación con la toma de nuevos estudios con contraste. A) diciembre 2007, B) marzo 2014, C) octubre 2016. 


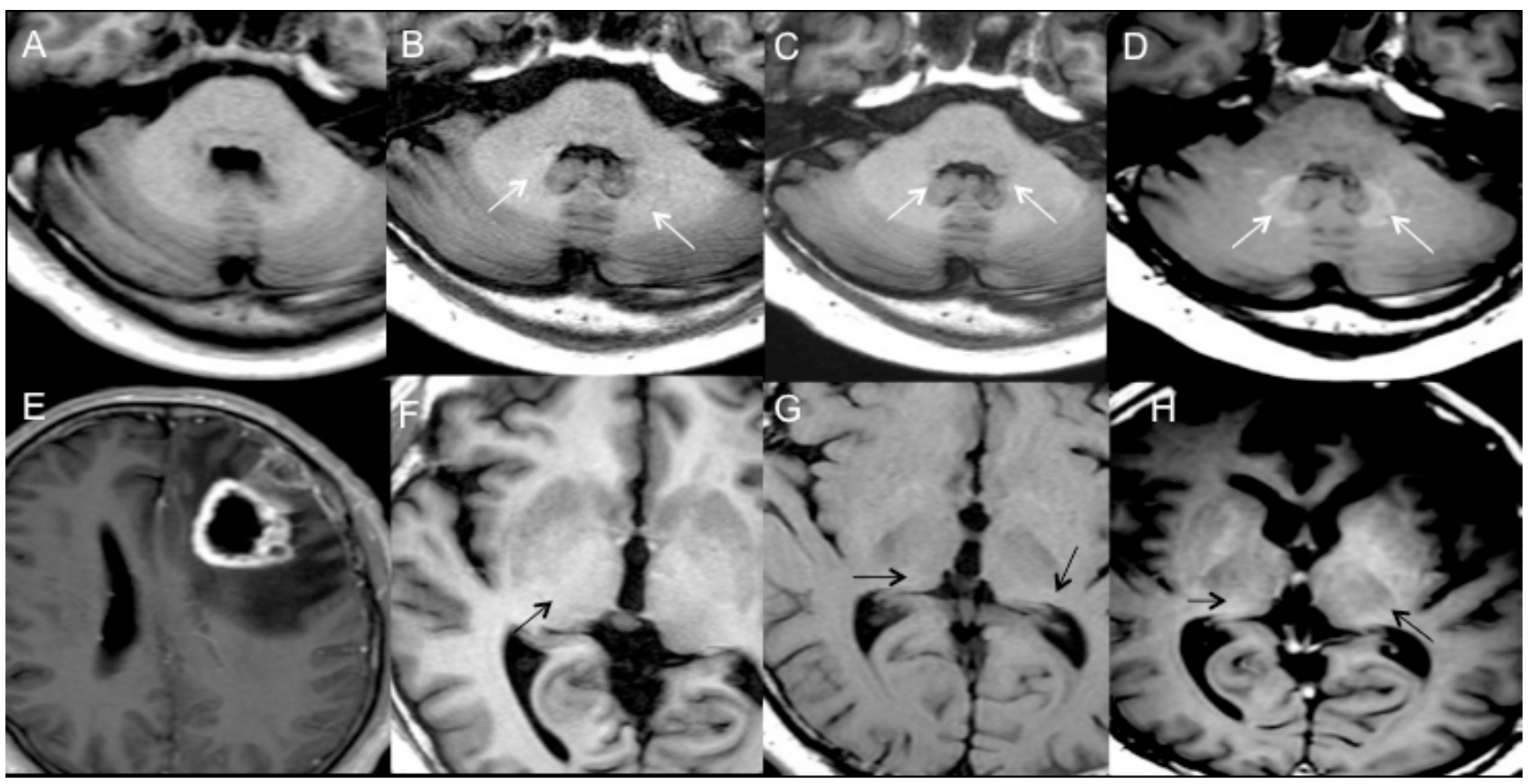

Figura 3. Imagen de resonancia magnética, T1, cortes axiales. Paciente en seguimiento por Glioblastoma, en quien se evidencia reforzamiento del núcleo dentado (flechas blancas) progresivo a través del tiempo, en relación con la toma de nuevos estudios con contraste. Adicionalmente se observa reforzamiento en los gánglios basales (flechas negras). A) año 2012, B) 2013, C) 2014, D) 2015, E) 2012, F) 2013, G) junio 2015, H) Noviembre 2015.

o no iónicos. Los MCBG macrocíclicos y los iónicos son los más estables, es decir, son los que tienen menos riesgo de disociarse y liberar el ion toxico de gadolinio. En los últimos dos años se han publicado múltiples investigaciones que muestran a través del estudio histológico y las imágenes de resonancia magnética el depósito de gadolinio en el núcleo dentado y el globo pálido, que es proporcional al número de dosis de gadolinio recibidas y es más marcado con el uso de MCBG lineales no iónicos como la Gadodiamida. El significado clínico del depósito de gadolinio en el sistema nervioso central permanece incierto hasta la fecha.

\section{Bibliografía}

1. Hao, D. et al. MRI contrast agents: basic chemistry and safety. J Magn Reso. Imaging JMRI 2012; 36: 1060-1071.

2. Rowley, H. A. et al. Contrast-enhanced MR imaging of brain lesions: a large-scale intraindividual crossover comparison of gadobenate dimeglumine versus gadodiamide. AJNR Am J Neuroradiol 2008; 29: 1684-1691.

3. Kanda, T., Oba, H., Toyoda, K., Kitajima, K. \& Furui, $\mathrm{S}$. Brain gadolinium deposition after administration of gadolinium-based contrast agents. Jpn J Radiol 2016; 34: 3-9.

4. Ramalho, J. et al. Gadolinium-Based Contrast Agent Accumulation and Toxicity: An Update. AJNR Am J Neuroradiol 2016; 37: 1192-1198.

5. Kanal, E. \& Tweedle, M. F. Residual or retained gado- linium: practical implications for radiologists and our patients. Radiology 2015; 275: 630-634.

6. Rocklage, S. M., Worah, D. \& Kim, S. H. Metal ion release from paramagnetic chelates: what is tolerable? Magn Reson Med 1991; 22: 216-221-232.

7. Grobner, T. Gadolinium-a specific trigger for the development of nephrogenic fibrosing dermopathy and nephrogenic systemic fibrosis? Nephrol. Dial. Transplant. Off. Publ. Eur. Dial. Transpl. Assoc. Eur Ren Assoc 2006; 21: 1104-1108.

8. Marckmann, P. et al. Nephrogenic systemic fibrosis: suspected causative role of gadodiamide used for contrast-enhanced magnetic resonance imaging. J. Am. Soc. Nephrol. JASN 2006; 17: 2359-2362.

9. Sadowski, E. A. et al. Nephrogenic systemic fibrosis: risk factors and incidence estimation. Radiology 2007; 243: 148-157.

10. Kanda, T., Ishii, K., Kawaguchi, H., Kitajima, K. \& Takenaka, D. High signal intensity in the dentate nucleus and globus pallidus on unenhanced T1-weighted MR images: relationship with increasing cumulative dose of a gadolinium-based contrast material. Radiology 2014; 270: 834-841.

11. Huckle, J. E., Altun, E., Jay, M. \& Semelka, R. C. Gadolinium Deposition in Humans: When Did We Learn That Gadolinium Was Deposited In Vivo? Invest. Radiol 2016; 51: 236-240.

12. Kanda, T. et al. High Signal Intensity in Dentate Nucleus on Unenhanced T1-weighted MR Images: Association with Linear versus Macrocyclic Gadolinium Chelate Administration. Radiology 2015; 275: 803-809.

13. Stojanov, D., Aracki-Trenkic, A. \& Benedeto-Stojanov, 
D. Gadolinium deposition within the dentate nucleus and globus pallidus after repeated administrations of gadolinium-based contrast agents-current status. Neuroradiology 2016; 58: 433-441.

14. Quattrocchi, C. C. et al. Gadodiamide and Dentate Nucleus T1 Hyperintensity in Patients With Meningioma Evaluated by Multiple Follow-Up Contrast-Enhanced Magnetic Resonance Examinations With No Systemic Interval Therapy. Invest. Radiol 2015; 50: 470-472.

15. McDonald, R. J. et al. Intracranial Gadolinium Deposition after Contrast-enhanced MR Imaging. Radiology 2015; 275: 772-782.

16. Kanda, T. et al. Gadolinium-based Contrast Agent Accumulates in the Brain Even in Subjects without Severe Renal Dysfunction: Evaluation of Autopsy Brain Specimens with Inductively Coupled Plasma Mass Spectroscopy. Radiology 2015; 276: 228-232.

17. Ramalho, J. et al. High Signal Intensity in Globus Pallidus and Dentate Nucleus on Unenhanced T1-weighted MR Images: Evaluation of Two Linear Gadolinium-based Contrast Agents. Radiology 2015; 276: 836-844.

18. Errante, Y. et al. Progressive increase of T1 signal intensity of the dentate nucleus on unenhanced magnetic resonance images is associated with cumulative doses of intravenously administered gadodiamide in patients with normal renal function, suggesting dechelation. Invest Radiol 2014; 49: 685-690.

19. Robert, P. et al. Linear Gadolinium-Based Contrast Agents Are Associated With Brain Gadolinium Retention in Healthy Rats. Invest. Radiol 2016; 51: 73-82.

20. Radbruch, A. et al. Gadolinium retention in the dentate nucleus and globus pallidus is dependent on the class of contrast agent. Radiology 2015; 275: 783-791.

21. Murata, N. et al. Macrocyclic and Other Non-Group 1 Gadolinium Contrast Agents Deposit Low Levels of Gadolinium in Brain and Bone Tissue: Preliminary Results From 9 Patients With Normal Renal Function. Invest. Radiol 2016; 51: 447-453.

22. Cao, Y. et al. Effect of Renal Function on GadoliniumRelated Signal Increases on Unenhanced T1-Weighted Brain Magnetic Resonance Imaging. Invest Radiol 2016. doi:10.1097/RLI.0000000000000294

23. Weberling, L. D. et al. Increased Signal Intensity in the Dentate Nucleus on Unenhanced T1-Weighted Images After Gadobenate Dimeglumine Administration. Invest Radiol 2015; 50: 743-748.

24. Hu, H. H., Pokorney, A., Towbin, R. B. \& Miller, J. H. Increased signal intensities in the dentate nucleus and globus pallidus on unenhanced T1-weighted images: evidence in children undergoing multiple gadolinium MRI exams. Pediatr Radiol 2016. doi:10.1007/s00247016-3646-3

25. Roberts, D. R. \& Holden, K. R. Progressive increase of T1 signal intensity in the dentate nucleus and globus pallidus on unenhanced T1-weighted MR images in the pediatric brain exposed to multiple doses of gadolinium contrast. Brain Dev 2016; 38: 331-336.

26. Khadilkar, S. et al. A practical approach to diseases affecting dentate nuclei. Clin Radiol 2016; 71: 107-119.

27. Kanda, T. et al. Contribution of metals to brain MR signal intensity: review articles. Jpn J Radiol 2016; 34: 258-266. 